\title{
Transverse spin densities of octet baryons using Lattice QCD
}

\author{
J. M. Bickerton ${ }^{a}$, R. Horsley ${ }^{b}$, Y. Nakamura ${ }^{c}$, P. E. L. Rakow ${ }^{d}$, G. Schierholz ${ }^{e}$ \\ P. E. Shanahan ${ }^{f}$, R. Young ${ }^{a, g}$, J. M. Zanotti ${ }^{*}, a$ \\ a CSSM, Department of Physics, The University of Adelaide, Adelaide SA 5005, Australia \\ ${ }^{b}$ School of Physics and Astronomy, University of Edinburgh, Edinburgh EH9 3FD, UK \\ ${ }^{c}$ RIKEN Advanced Institute for Computational Science, Kobe, Hyogo 650-0047, Japan \\ ${ }^{d}$ Theoretical Physics Division, Department of Mathematical Sciences, University of Liverpool, \\ Liverpool L69 3BX, UK \\ e Deutsches Elektronen-Synchrotron DESY, 22603 Hamburg, Germany \\ ${ }^{f}$ Center for Theoretical Physics, Massachusetts Institute of Technology, Cambridge, MA 02139, \\ U.S.A. \\ ${ }^{g}$ CoEPP, Department of Physics, The University of Adelaide, Adelaide SA 5005, Australia \\ E-mail: \\ jacob.bickerton@adelaide.edu.au, \\ james.zanotti@adelaide.edu.au
}

\section{QCDSF/UKQCD Collaboration}

We present results from the QCDSF/UKQCD collaboration for the transverse spin densities of octet baryons obtained from simulations using $N_{f}=2+1$ flavours of $\mathscr{O}(a)$-improved Wilson fermions. These densities are revealed through an analysis of the electromagnetic and tensor form factors of the octet baryons at two different lattice spacings with pion masses as low as $220 \mathrm{MeV}$. We find SU(3) flavour-breaking effects of the form factors and use these to extrapolate to the physical mass. Constructing combinations of Fourier transformed form factors reveal non-trivial spin densities in the transverse plane, with similar deformations across the baryon octet.

34th annual International Symposium on Lattice Field Theory

24-30 July 2016

University of Southampton, UK

\footnotetext{
* Speaker.
} 


\section{Introduction}

A full understanding of how the nucleon and other hadrons are constructed from their quark and gluon constituents remains one of the most important and challenging questions in modern nuclear physics. Thanks to their probability density interpretation, generalised parton distributions (GPDs) in impact parameter space [1] are an extremely valuable tool for achieving this goal. By determining the lowest few moments of GPDs, lattice QCD simulations have already provided valuable insights into the transverse spin structure of the nucleon [2]. This is achieved through the definition of the $x^{n-1}$ moment of the density of transversely polarised quarks (with spin vector $s_{\perp}$ ) in a transversely polarised nucleon (with spin vector $S_{\perp}$ ) at impact parameter $b_{\perp}$ [3],

$$
\begin{aligned}
\rho^{n}\left(b_{\perp}, s_{\perp}, S_{\perp}\right) & =\int_{-1}^{1} d x x^{n-1} \rho\left(x, b_{\perp}, s_{\perp}, S_{\perp}\right) \\
& =\frac{1}{2}\left\{A_{n 0}\left(b_{\perp}^{2}\right)+s_{\perp}^{i} S_{\perp}^{i}\left(A_{T n 0}\left(b_{\perp}^{2}\right)-\frac{1}{4 m^{2}} \triangle_{b_{\perp}} \tilde{A}_{T n 0}\left(b_{\perp}^{2}\right)\right)\right. \\
& \left.+\frac{b_{\perp}^{j} \mathcal{E}^{j i}}{m}\left(S_{\perp}^{i} B_{n 0}^{\prime}\left(b_{\perp}^{2}\right)+s_{\perp}^{i} \bar{B}_{T n 0}^{\prime}\left(b_{\perp}^{2}\right)\right)+s_{\perp}^{i}\left(2 b_{\perp}^{i} b_{\perp}^{j}-b_{\perp}^{2} \delta^{i j}\right) S_{\perp}^{j} \frac{1}{m^{2}} \tilde{A}_{T n 0}^{\prime \prime}\left(b_{\perp}^{2}\right)\right\} .
\end{aligned}
$$

The impact parameter dependent generalised form factors (GFFs) $A_{n 0}\left({ }_{\perp}^{2}\right), A_{T n 0}\left(b_{\perp}^{2}\right), \ldots$ in Eq. (1.1) are related to the momentum space GFFs $A_{n 0}(t), A_{T n 0}(t), \ldots$ by a Fourier transform

$$
f\left(b_{\perp}^{2}\right) \equiv \int \frac{d^{2} \Delta_{\perp}}{(2 \pi)^{2}} e^{-i b_{\perp} \cdot \Delta_{\perp}} f\left(t=-\Delta_{\perp}^{2}\right),
$$

where we denote the momentum transfered to the hadron by $\Delta$ with $t=\Delta^{2}$. The derivatives of the impact parameter GFFs in Eq. (1.1) are defined as $f^{\prime} \equiv \partial_{b_{\perp}^{2}} f$ and $\triangle_{b_{\perp}} f \equiv 4 \partial_{b_{\perp}}\left(b_{\perp}^{2} \partial_{b_{\perp}}\right) f$.

The QCDSF/UKQCD collaboration has embarked on a program to systematically investigate the role of $S U$ (3)-flavour symmetry breaking in hadron properties $[4,5,6,7,8,9,10,11]$. In this talk we extend this program and present preliminary $N_{f}=2+1$ lattice QCD results for the octet baryon electromagnetic and tensor form factors, and how they can lead to a determination of the first $(n=1)$ moment of their transverse spin densities.

\section{Simulation Details}

Our gauge field configurations have been generated with $N_{f}=2+1$ flavours of dynamical fermions, using the tree-level Symanzik improved gluon action and nonperturbatively $\mathscr{O}(a)$ improved Wilson fermions [12]. We choose our quark masses by first finding the $S U(3)_{\text {flavour }}{ }^{-}$ symmetric point where flavour singlet quantities take on their physical values, then varying the individual quark masses while keeping the singlet quark mass $\bar{m}_{q}=\left(m_{u}+m_{d}+m_{s}\right) / 3=\left(2 m_{l}+m_{s}\right) / 3$ constant [4]. We have generated a large set of ensembles of varying quark masses and volumes at $\beta=5.50$ and $\beta=5.80$, corresponding to lattice spacings, $a=0.074 \mathrm{fm}$ and $a=0.059 \mathrm{fm}$ respectively, where we have used a variety of singlet quantities to set the scale $[4,5,13]$. The results presented in this proceedings are obtained on a subset of the complete set of ensembles [13], with the range displayed in Fig. 1 of [6]. More details regarding the tuning of our simulation parameters are given in Refs. [4, 5, 13]. 


\section{Electromagnetic and Tensor Form Factors}

In order to determine the lowest $(n=1)$ moment of the transverse spin densities of the octet baryons, we require lattice QCD determinations of the electromagnetic and tensor form factors.

On the lattice, we determine the Dirac $F_{1}(t)=A_{10}(t)$ and Paul $F_{2}(t)=A_{20}(t)$ form factors by calculating the following matrix element of the electromagnetic current

$$
\left\langle B\left(p^{\prime}, s^{\prime}\right)\left|j_{\mu}(\Delta)\right| B(p, s)\right\rangle=\bar{u}\left(p^{\prime}, s^{\prime}\right)\left[\gamma_{\mu} F_{1}(t)+\sigma_{\mu v} \frac{\Delta_{v}}{2 M_{B}} F_{2}(t)\right] u(p, s),
$$

where $u(p, s)$ is a Dirac spinor with momentum $p$, and spin polarisation $s, \Delta=p^{\prime}-p$ is the momentum transfer, $M_{B}$ is the mass of the baryon $B$, and $j_{\mu}$ is the electromagnetic current. The form factors of the proton are obtained by using $j_{\mu}^{(p)}=\frac{2}{3} \bar{u} \gamma_{\mu} u-\frac{1}{3} \bar{d} \gamma_{\mu} d$, while the form factors for the $\Sigma$ and $\Xi$ baryons are obtained through the appropriate substitution, $u \rightarrow s$ or $d \rightarrow s$. Here we will be focussing on quark flavour distributions so we will consider only the individual quark contributions to the total baryon form factors.

The transverse density also requires the tensor form factors of the octet baryons,

$$
\left\langle B\left(p^{\prime}, s^{\prime}\right)\left|\mathscr{O}_{T}^{\mu v}(\Delta)\right| B(p, s)\right\rangle=\bar{u}\left(p^{\prime}, s^{\prime}\right)\left\{i \sigma^{\mu v} A_{T 10}(t)+\frac{\bar{P}^{[\mu} \Delta^{v]}}{M_{B}^{2}} \tilde{A}_{T 10}(t)+\frac{\gamma^{[\mu} \bar{P}^{v]}}{2 M_{B}} B_{T 10}(t)\right\} u(p, s),
$$

where $\mathscr{O}_{T}^{\mu v}=\bar{q} i \sigma^{\mu v} q$ is the tensor current for quark of flavour $q, \gamma^{[\mu} \bar{P}^{v]} \equiv \gamma^{\mu} \bar{P}^{v}-\gamma^{v} \bar{P}^{\mu}, \bar{P}=\frac{p^{\prime}+p}{2}$. We note that the value of the form factor $A_{T 10}$ at zero momentum transfer is the familiar tensor charge $A_{T 10}(t=0)=g_{T}$.

The electromagnetic form factors from these ensembles have appeared previously in [6, 7]. As an example of the quality of our form factors, in Fig. 1 we show the individual quark contributions to the electromagnetic form factors of the proton, $\Sigma$ and $\Xi$ baryons at a pion mass on $m_{\pi} \approx 310 \mathrm{MeV}$. In this preliminary work we perform dipole fits $F(t)=F(0) /\left(1-t / M^{2}\right)^{2}$ to $F_{1}$ and $F_{2}$ as seen in Fig. 1, although it is common knowledge that such an ansatz gives a poor description of the form factors. This will be addressed in future work. Similarly, Fig. 2 shows an example of the individual quark contributions to the octet baryon tensor form factors $A_{T 10}(t)$, where again, in this preliminary work these form factors are fitted using a dipole form.

\section{SU(3) Flavour Symmetry Breaking Extrapolation to Physical Mass}

To extrapolate to the physical quark masses, we consider an $S U(3)$ flavour symmetry breaking expansion [14] at fixed values of $Q^{2}=-t$ [6]. We proceed by constructing combinations of baryon form factors to form $f$-fan and $d$-fan plots [14], where the $x$-axis represents the pion mass of each configuration divided by a normalisation $X_{\pi}^{2}=\left(2 M_{K}^{2}+M_{\pi}^{2}\right) / 3$ such that the symmetric points all lie at $x=1$. Similarly we to construct the $y$-axis such that the form factor value of the symmertic points all lie at $y=1$. This is achieved by normalising the form factors at fixed momentum transfer by a flavour singlet quantity $X_{T}$ [14]. An example of an $f$-fan plot is shown in Fig. 3, for the first tensor form factor $A_{T 10}$ or $g_{T}$ at zero momentum transfer $\left(t=Q^{2}=0\right)$, i.e. this is the $f$-fan of the tensor charge. In the remainder of this proceedings we will focus on $f$-fan results (which correspond to doubly-represented quarks) and leave the $d$-fan results for a future publication. A 

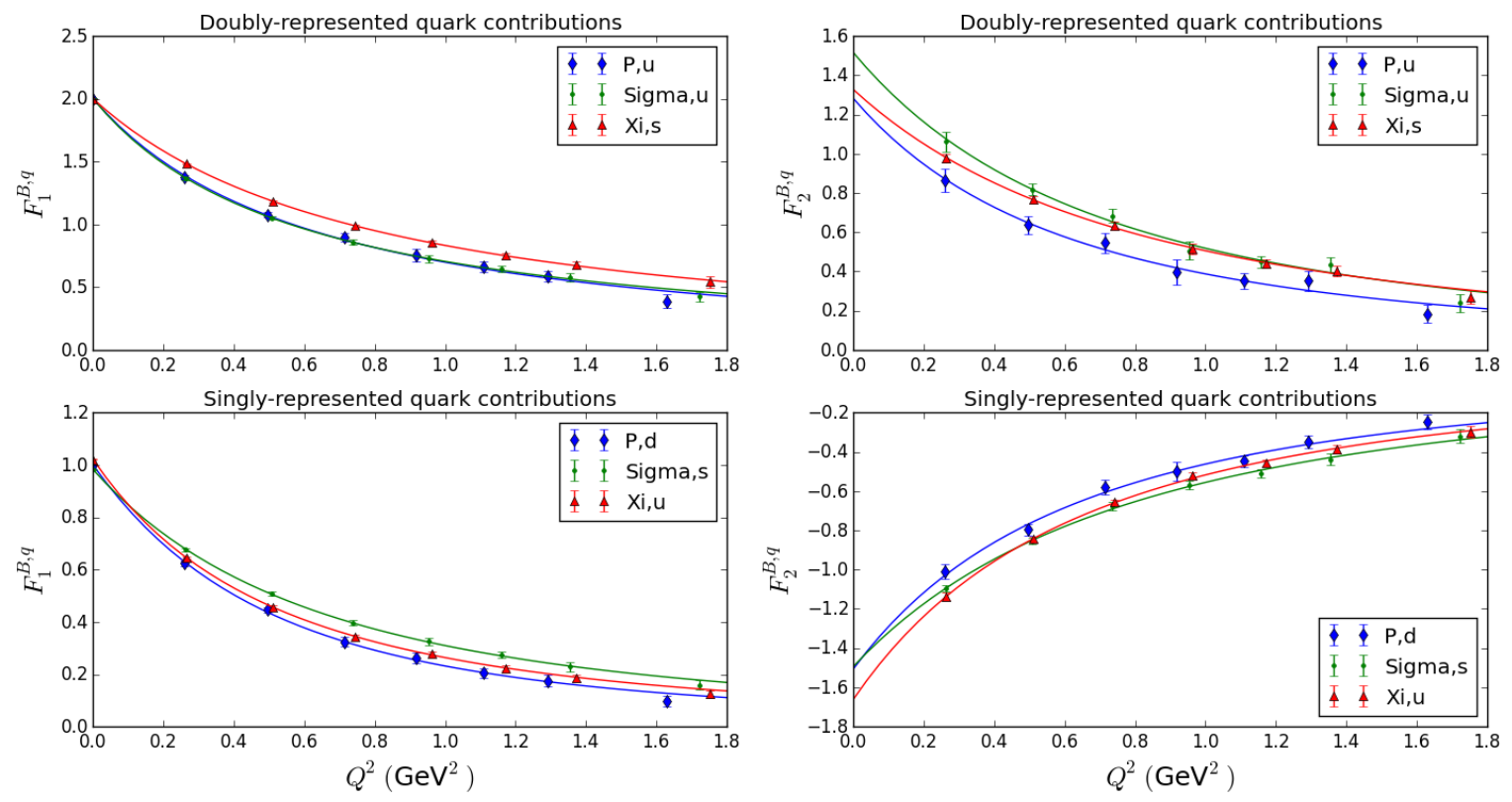

Figure 1: Individual quark contributions to the electromagnetic form factors of the proton, $\Sigma$ and $\Xi$ baryons at a pion mass on $m_{\pi} \approx 310 \mathrm{MeV}$, plotted as a function of $Q^{2}=-t$.

dotted black line represents the physical value of $m_{\pi}^{2} / X_{\pi}^{2}$, and thus each of the coloured stars represents our extrapolated value of the form factor value at that specific $Q^{2}$ for each baryon. The point that we see for the physical value is normalised by flavour singlet combination $X_{T}$. Since the tensor form factors of the octet baryons are not yet known experimentally, we determine $X_{T}$ at the physical mass by extrapolating our lattice values with a linear form as shown in Fig. 4. Since this is an $S U$ (3) flavour singlet, it should have very little dependence on the amount of $S U(3)$ flavour breaking (occurs at $\mathscr{O}\left((\delta m)^{2}\right)$ ) [5] and this is observed by the mild slope in our fitted results.

We repeat this method by first binning our results across all our ensembles into $6 t=Q^{2}$ bins (see Fig. 6 of [6]) and performing the $S U(3)$ flavour breaking expansion of the form factors for each of these bins. In Fig. 5 we present our results for the doubly-represented quark contribtions to the baryon tensor form factors $A_{T 10}(t)$ extrapolated to the physical masses as a function of $t=Q^{2}$ together with a dipole fit.

\section{Transverse Spin Densities}

Having now determined the individual quark contributions to the octet baryon EM and tensor form factors at the physical point, we are now in a position to determine the transverse spin densities of quarks inside the octet baryon as given in Eq. (1.1). As described in the previous section, in this preliminary study all form factors have been fitted using a dipole ansatz. We Fourier transform each dipole fit (Eq. (1.2)) to obtain impact parameter form factors. Finally, we construct a combination of two of the tensor form factors calculated in the previous section to form the third form factor that we require to calculate the transverse spin density, where $\bar{B}_{T 10}\left(Q^{2}\right)=2 \tilde{A}_{T 10}+B_{T 10}$, [3]. 

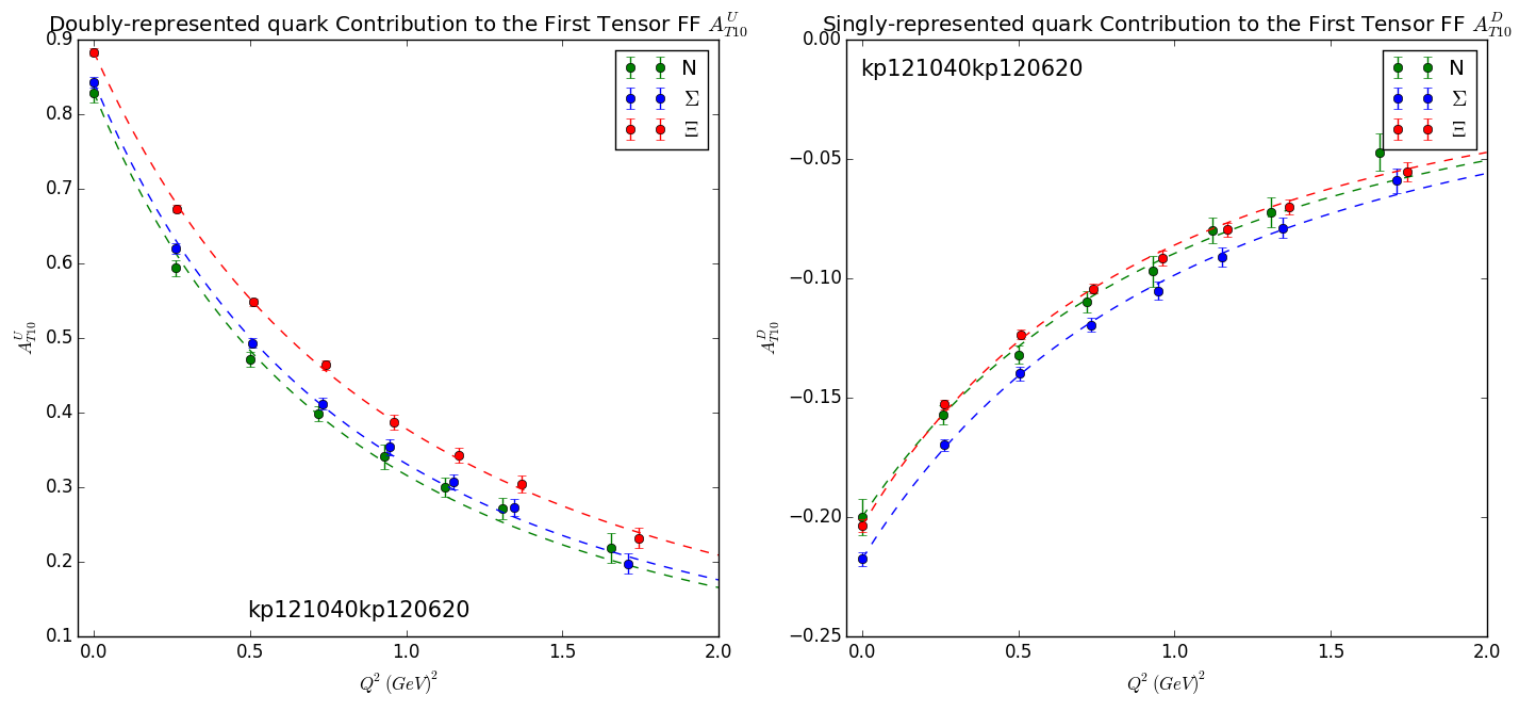

Figure 2: Individual quark contributions to the octet baryon tensor form factors together with dipole fits, plotted as a function of $Q^{2}=-t$.

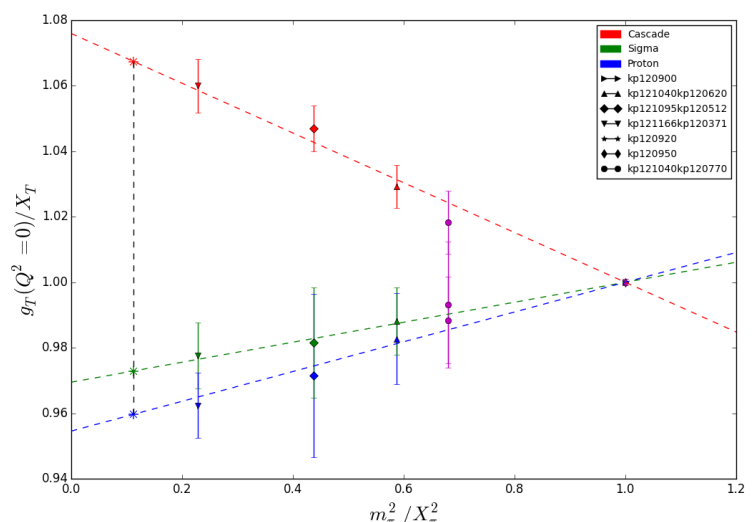

Figure 3: $f$-fan of $A_{T 10}=g_{T}$ at fixed $t=Q^{2}=0$ showing the extrapolation to the physical point.

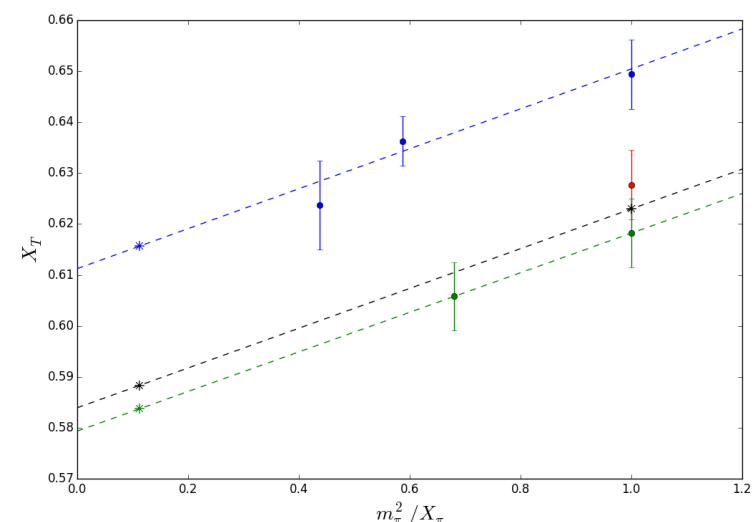

Figure 4: Linear extrapolation of flavour singlet $X_{T}$ for the $f$-fan for $A_{T 10}=g_{T}$ at $t=Q^{2}=0$.

If we analyse equation (1.1) in further detail we recognise the orbitally-symmetric monopole terms and further two dipole structures $b_{\perp}^{j} \varepsilon^{j i} S_{\perp}^{i}$ and $b_{\perp}^{j} \varepsilon^{j i} s_{\perp}^{i}$, and the last term corresponds to a quadrupole structure [2]. Thus the derivatives of the EM and tensor form factors $B_{10}\left(b_{\perp}\right), \bar{B}_{T 10}\left(b_{\perp}\right)$ and $\tilde{A}_{T 10}\left(b_{\perp}\right)$ all correspond to the strength of the distortion in the orbital symmetry in the transverse plane.

Choosing both $S_{\perp}^{i}, s_{\perp}^{i}=0$, isolates the situation where both the nucleon and quark spin are unpolarised. In this scenario, the only remaining term in the spin density equation (1.1) is the $\frac{1}{2} A_{10}\left(b_{\perp}\right)$ term and hence there is no distortion in the orbital symmetry, producing spherically symmetric quark distributions in the transverse (impact-parameter) plane.

In these proceedings, we choose to present as an example the distributions of unpolarised 


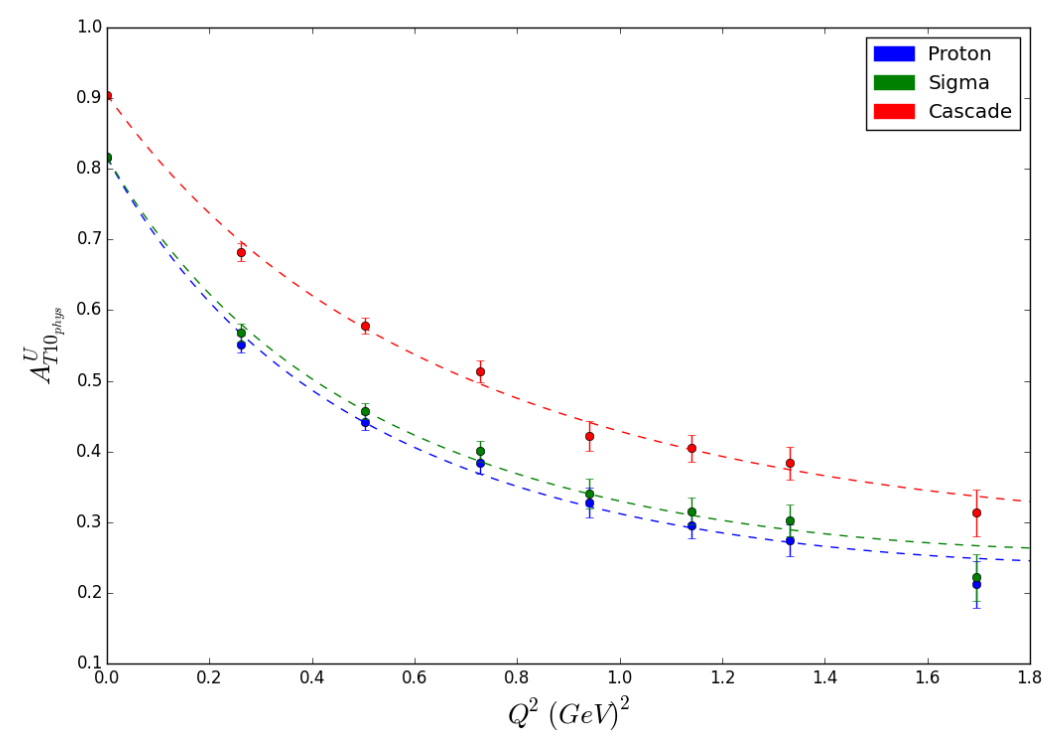

Figure 5: Tensor form factor $A_{T 10}$ extrapolated to the physical masses together with a dipole fit.

quarks in a baryon polarised in the $+x$-direction. The results for the $u$-quark in the proton and the $\Sigma$ baryon are compared in Fig. 6 where we observe a clear distortion from a spherically-symmetric behaviour due to the presence of a non-vanishing anomalous magnetic moment. While we see similarities in these doubly represented up quarks distributions, we note that distribtion of the $u$ quark in the $\Sigma$ is broader than that for the same flavour quark in the proton. This can only be due to the influence of the heavier spectator $s$-quark in the $\Sigma$ baryon as compared to the light spectator $d$-quark in the proton. Further effects of the quark spin distributions in the various baryons will be presented in a forthcoming publication.

\section{Acknowledgements}

The numerical configuration generation (using the BQCD lattice QCD program [15]) and data analysis (using the Chroma software library [16]) was carried out on the IBM BlueGene/Qs using DIRAC 2 resources (EPCC, Edinburgh, UK), and at NIC (Jülich, Germany) and the SGI ICE 8200 and Cray XC30 at HLRN (The North-German Supercomputer Alliance) and on the NCI National Facility in Canberra, Australia (supported by the Australian Commonwealth Government). This investigation has been supported in part by DFG Grant No. SCHI 179/8-1, STFC under contract ST/G00062X/1 and the Australian Research Council under grants FT120100821, FT100100005, DP150103164, DP140103067 and CE110001004. We thank all funding agencies.

\section{References}

[1] M. Burkardt, Phys. Rev. D 62 (2000) 071503 Erratum: [Phys. Rev. D 66 (2002) 119903] [hep-ph/0005108].

[2] M. Gockeler et al.,Phys. Rev. Lett. 98, 222001 (2007) [hep-lat/0612032]. 
Proton

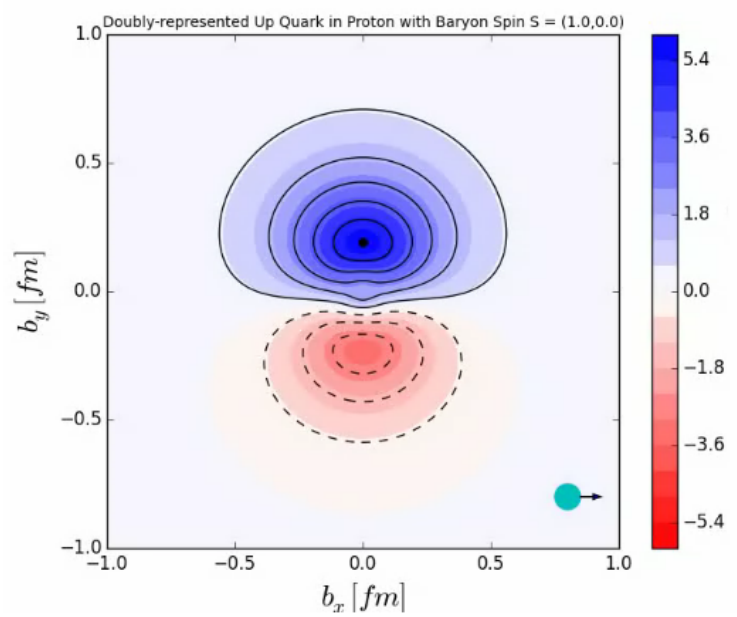

Sigma

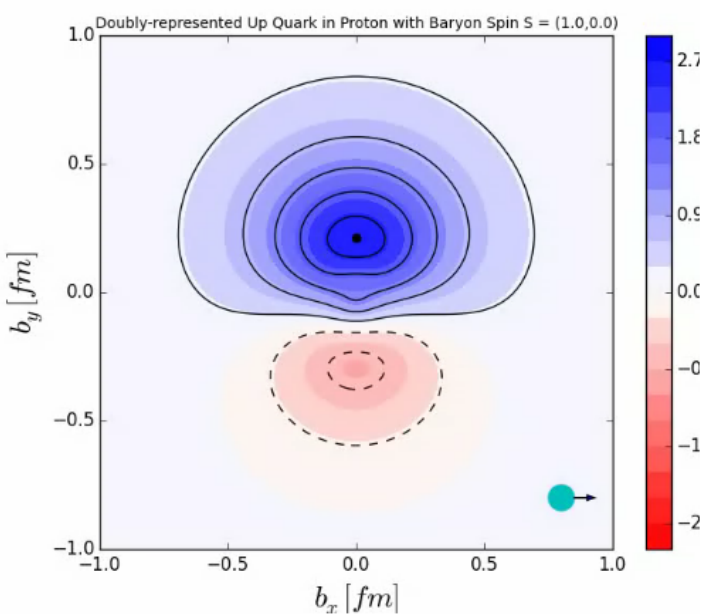

Figure 6: Spin densities distribution of an unpolarised $u$-quark in a transversely polarised nucleon (left) and $\Sigma$ baryon (right).

[3] M. Diehl and P. Hagler, Eur. Phys. J. C 44, 87 (2005) [hep-ph/0504175].

[4] W. Bietenholz et al.,Phys. Lett. B690 (2010) 436 [1003.1114 [hep-lat]].

[5] W. Bietenholz et al.,Phys. Rev. D 84 (2011) 054509 [1102.5300 [hep-lat]].

[6] P. E. Shanahan et al.,Phys. Rev. D 89 (2014) 074511 [arXiv:1401.5862 [hep-lat]].

[7] P. E. Shanahan et al., Phys. Rev. D 90 (2014) 034502 [arXiv:1403.1965 [hep-lat]].

[8] R. Horsley et al., Phys. Rev. D 91 (2015) no.7, 074512 [arXiv:1411.7665 [hep-lat]].

[9] P. E. Shanahan et al., Phys. Rev. D 91 (2015) no.11, 113006 [arXiv:1503.01142 [hep-lat]].

[10] P. E. Shanahan et al., Phys. Rev. D 92 (2015) no.7, 074029 [arXiv:1508.06923 [nucl-th]].

[11] V. G. Bornyakov et al., arXiv:1612.04798 [hep-lat].

[12] N. Cundy et al.,Phys. Rev. D79 (2009) 094507 [0901.3302 [hep-lat]].

[13] V. G. Bornyakov et al., arXiv:1508.05916 [hep-lat].

[14] A. N. Cooke et al., PoS LATTICE 2012, 116 (2012) [arXiv:1212.2564 [hep-lat]].

[15] Y. Nakamura and H. Stüben, PoS LATTICE 2010 (2010) 040 [1011.0199 [hep-lat]].

[16] R. G. Edwards and B. Joó, Nucl. Phys. Proc. Suppl. 140 (2005) 832 [hep-lat/0409003]. 